\title{
Refractive index modulation based on excitonic effects in GalnAs-InP coupled asymmetric quantum wells
}

Thirstrup, Carsten

Published in:

I E E E Journal of Quantum Electronics

Link to article, DOI:

10.1109/3.387034

Publication date:

1995

Document Version

Publisher's PDF, also known as Version of record

Link back to DTU Orbit

Citation (APA):

Thirstrup, C. (1995). Refractive index modulation based on excitonic effects in GalnAs-InP coupled asymmetric quantum wells. I E E E Journal of Quantum Electronics, 31(6), 988-996. https://doi.org/10.1109/3.387034

\section{General rights}

Copyright and moral rights for the publications made accessible in the public portal are retained by the authors and/or other copyright owners and it is a condition of accessing publications that users recognise and abide by the legal requirements associated with these rights.

- Users may download and print one copy of any publication from the public portal for the purpose of private study or research.

- You may not further distribute the material or use it for any profit-making activity or commercial gain

- You may freely distribute the URL identifying the publication in the public portal

If you believe that this document breaches copyright please contact us providing details, and we will remove access to the work immediately and investigate your claim 


\title{
Refractive Index Modulation Based on Excitonic Effects in GaInAs-InP Coupled Asymmetric Quantum Wells
}

\author{
Carsten Thirstrup
}

\begin{abstract}
The effect of excitons in GaInAs-InP coupled asymmetric quantum wells on the refractive index modulation, is analyzed numerically using a model based on the effective mass approximation. It is shown that two coupled quantum wells brought in resonance by an applied electric field will, due to the reduction in the exciton oscillator strengths, have a modulation of the refractive index which is more than one order of magnitude larger than in a similar quantum well structure based on the quantum confined Stark effect, but with no coupling between the quantum wells. Calculations show that combining this strong electrorefractive effect with self-photo-induced modulation in a biased-pin-diode modulator configuration, results in an optical nonlinearity with a figure of merit of $20 \mathrm{~cm}^{3} / J$ at a wavelength of $1.55 \mu \mathrm{m}$. This value is large compared to optical nonlinearities originating from band edge resonance effects in III-V semiconductor materials.
\end{abstract}

\section{INTRODUCTION}

I NTERFEROMETRIC devices based on a modulation of the refractive index are attractive for monolithic integration with semiconductor lasers [1]. For such devices, it is desirable to obtain maximum refractive index changes and minimum absorption losses. In multiple quantum well (MQW) structures, it has been shown by Zucker et al. [2] that a large refractive index modulation can be achieved close to the absorption edge due to the quantum confined Stark effect (QCSE). For waveguide applications, however, the operating wavelength needs to be sufficiently below the absorption edge where the change in the refractive index has decreased significantly. Carrier effects do not suffer from the same limitations, and larger refractive index modulation at similar absorption losses have been obtained due to the bandfilling effect in a barrier, reservoir, and quantum well electron transfer device [1], [3]. On the other hand, it has been shown theoretically that for a three-step asymmetric coupled quantum well structure, the QCSE yields an enhancement in the refractive index modulation by approximately one order of magnitude, compared to the case for a conventional MQW structure [4].

Another possibility is to use the effect of excitons in coupled quantum wells. Fox et al. [5]-[7] has investigated this effect in GaAs-GaAlAs symmetric quantum wells (QW's), each with two confined states per QW, the coupling being between the ground state in one $\mathrm{QW}$, and the first excited state in the neighboring QW. This paper focuses, theoretically, on the

Manuscript received March 21, 1994; revised November 15, 1994.

The author is with the Mikroelektronik Centret, Technical University of Denmark, Building 345E, 2800 Lyngby, Denmark.

IEEE Log Number 9410760 effect of excitons in GaInAs-InP asymmetric QW's on the refractive index modulation, where the coupling is between the two ground states in two neighboring QW's. A numerical model, which takes account of the effect of the Coulomb mixing of the excitons shows that in a structure comprising a coupled QW-structure involving a $30-\AA$ and a $40-\AA$ GaInAs QW, separated by a $50-\AA \AA$ InP barrier, the electric field induced refractive index change is more than one order of magnitude larger than in a structure comprising the same QW's, but with no coupling between the QW's. The QW's are narrow resulting in a negligible QCSE.

Compared to the QCSE, the change in refractive index due to excitons in GaInAs-InP coupled asymmetric QW's is qualitatively different. Below the absorption edge where the optical losses are low, the refractive index change $\Delta n$ decreases with increasing electric field until the electron wavefunctions from the two QW's are in resonance, whereupon $\Delta n$ increases again and approaches zero at large electric fields $(>$ $70 \mathrm{kV} / \mathrm{cm}$ ).

The large electrorefractive effect can appropriately be utilized in devices based on nipi-structures [8] or biased-pindiode structures, where photogenerated carriers induce a modulation of the electric fields across the $i$-regions comprising the QW's. From this self-photo-induced refractive index modulation, an optical nonlinearity can be defined, and simple estimations show that for a biased-pin-diode configuration, the figure of merit of the nonlinearity is more than one order of magnitude larger than band edge resonance effects in III-V semiconductor materials. The mechanism is similar to that in a self electrooptic effect device [9], which is based on a nonlinear modulation of the absorption coefficient rather than a modulation of the refractive index. There is no feedback as in the SEED, however.

This paper is organized as follows: Section II outlines under which conditions large refractive index changes can be achieved below the absorption edge. A qualitative description of the physical effect of excitons in coupled asymmetric quantum wells is presented in Section III, and in Section IV, a model of the effect is described. In Section V, numerical results of the refractive index modulation due to excitons in GaInAs-InP coupled QW's are shown, and an analysis of the optical nonlinearity based on the proposed structure and self-photo-induced modulation is presented in Section VI. Finally, in Section VI, the results of the paper are concluded. 


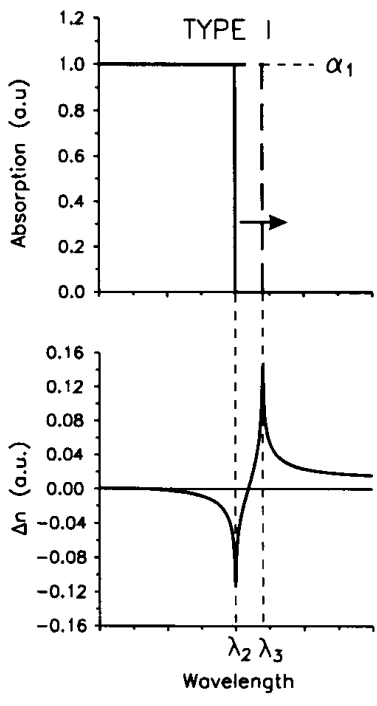

(a)

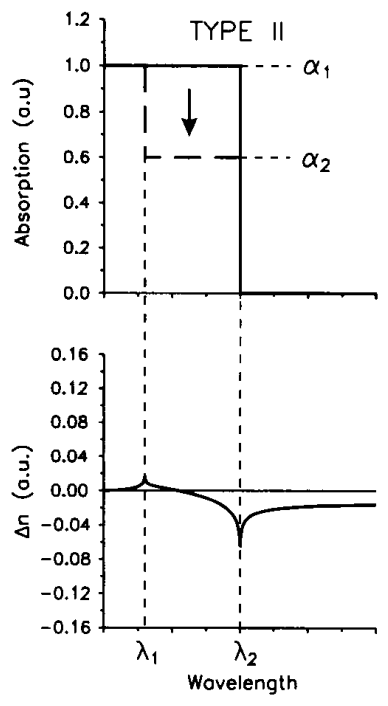

(b)

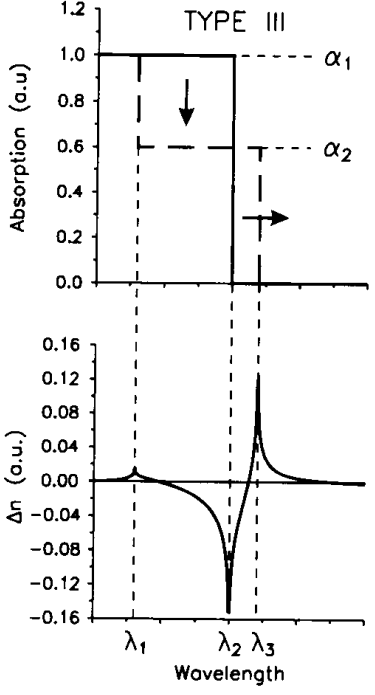

(c)

Fig. 1. Effect of Kramers-Kronig transform on three simple cases of change in the absorption coefficient. The top of the figure shows the absorption coefficient in the case of no excitation (solid curve) and with excitation (dashed curve) of the material; the arrows indicating the direction of the change. The bottom of the figure shows the corresponding change in the refractive index. In (a) the absorption edge is displaced from wavelength $\lambda_{2}$ to $\lambda_{3}$ (Type I effect); in (b) the absorption is reduced from $\alpha_{1}$ to $\alpha_{2}$ in the wavelength regime from $\lambda_{1}$ to $\lambda_{2}$ (Type II effect); and in (c) the two effects from (a) and (b) occur at the same time (Type III effect).

\section{ReFRaCtive INDEX MOdULATION}

The relationship between change in absorption $\Delta \alpha$ and change in refractive index $\Delta n$ is given by the Kramers-Kronig relation (e.g., [10])

$$
\Delta n(\lambda)=\frac{\lambda^{2}}{2 \pi^{2}} P \int \frac{\Delta \alpha\left(\lambda^{\prime}\right)}{\lambda^{2}-\lambda^{\prime 2}} d \lambda^{\prime}
$$

where $\lambda$ is the wavelength of light and $P$ denotes the principle value of the integral.

Fig. 1 illustrates the effect of the Kramers-Kronig transform on three simple cases of change in the absorption coefficient of materials with particular characteristics. The top of Fig. 1 shows the absorption coefficient in the case of no excitation of the material (solid curve) and in the case of excitation of the material (dashed curve). The arrows indicate the direction of the change. The bottom of Fig. 1 shows the corresponding changes in the refractive index $\Delta n$ when the material is excited. In the case shown in Fig. 1(a), the absorption edge is displaced from a wavelength $\lambda_{2}$ to a longer wavelength $\lambda_{3}$ (Type I effect); in Fig. 1(b), the absorption is reduced from $\alpha_{1}$ to $\alpha_{2}$ in the wavelength regime from $\lambda_{1}$ to $\lambda_{2}$ near the absorption edge (Type II effect); and in Fig. 1(c), the two effects from Fig. 1(a) and (b) occur at the same time (Type III effect).

In an optical phase modulator based on a waveguide configuration, it is desirable to obtain maximum change in the refractive index with minimum absorption losses. The operating wavelength should, therefore, be larger than $\lambda>\lambda_{3}$ in Fig. 1. For the cases shown in Fig. 1, simple analytical expressions for $\Delta n$ can be obtained and for wavelengths $\lambda>\lambda_{3}$, the results are
Type I:

$$
\Delta n_{1}(\lambda)=\frac{\lambda}{4 \pi^{2}} \alpha_{1} \ln \left\{\frac{\left(\lambda+\lambda_{3}\right)\left(\lambda-\lambda_{2}\right)}{\left(\lambda-\lambda_{3}\right)\left(\lambda+\lambda_{2}\right)}\right\}
$$

Type II:

$$
\Delta n_{\mathrm{II}}(\lambda)=\frac{-\lambda}{4 \pi^{2}}\left(\alpha_{1}-\alpha_{2}\right) \ln \left\{\frac{\left(\lambda+\lambda_{2}\right)\left(\lambda-\lambda_{1}\right)}{\left(\lambda-\lambda_{2}\right)\left(\lambda+\lambda_{1}\right)}\right\}
$$

Type III:

$$
\Delta n_{\mathrm{III}}(\lambda)=\Delta n_{\mathrm{I}}(\lambda)+\Delta n_{\mathrm{II}}(\lambda) .
$$

It is noted from (2) and Fig. 1, that a Type I effect yields a positive change in the refractive index below the absorption edge, and a Type II effect yields a negative change. The Type III effect is a sum of these two effects and, depending on whether the shift in the absorption edge or the reduction in the absorption dominates, the effect can be either positive, negative, or zero. Close to the absorption edge $\left(\lambda=\lambda_{2}\right)$ Type I and Type II effects have the same sign, and the refractive index modulation is enlarged for a Type III effect. In a waveguide configuration, however, the operating wavelength $\lambda>\lambda_{3}$, implying that $\Delta n_{\mathrm{I}}(\lambda)$ and $\Delta n_{\mathrm{II}}(\lambda)$ have opposite signs and tend to cancel each other (see (2) and Fig. 1(c)).

The QCSE involves a combined shift in the absorption edge and a decrease in the oscillator strengths near the absorption edge, so this effect has the features of Type III. The bandfilling effect has the features of Type II and has proven to yield a stronger electrorefractive effect than the QCSE [3]. As it will be shown in the following, a Type II effect can also be obtained by utilizing the effect of reducing the exciton binding energies in narrow GaInAs-InP coupled asymmetric quantum wells, where the QCSE is negligible. 


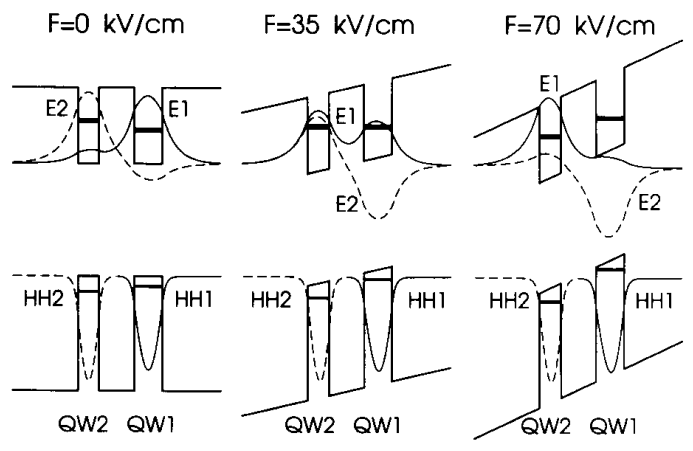

(a)

(b)

(c)

Fig. 2. Schematics of the energy bands of a coupled quantum well structure with a $30-\AA$ GaInAs quantum well (QW2), and a $40-\AA$ GaInAs quantum well (QW1) separated by a $50-\AA$ InP barrier in the case of an applied electric field of (a) $F=0 \mathrm{kV} / \mathrm{cm}$, (b) $F=35 \mathrm{kV} / \mathrm{cm}$, and (c) $F=70 \mathrm{kV} / \mathrm{cm}$. The position of the first electron energy subbands $E 1, E 2$ and the first heavy hole subbands $\mathrm{HH} 1, \mathrm{HH} 2$ are indicated with bold solid lines. The corresponding electron and hole wavefunctions are plotted with solid curves for the states confined to QW1 at zero electric field and dashed curves for the states confined to QW2 at zero electric field.

\section{EXCITONS IN GaInAs-InP COUPLED ASYMMETRIC QUANTUM WELLS}

Fig. 2 shows schematically the energy bands of a $30-\AA$ GaInAs QW and a $40-\AA$ GaInAs QW separated by a $50-\AA$ InP barrier in the case of an applied electric field of (a) $F=0$ $\mathrm{kV} / \mathrm{cm}$, (b) $F=35 \mathrm{kV} / \mathrm{cm}$, and (c) $F=70 \mathrm{kV} / \mathrm{cm}$. The two QW's can resonantly couple to each other. At zero electric field, the configuration allows two electron subband states (electron subband $E 1$ for the $40-\AA$ quantum well indicated QW1, and electron subband $E 2$ for the $30-\AA$ quantum well indicated QW2), 8 heavy hole subband states, and 3 light hole subband states (the first two heavy hole subband states are indicated $\mathrm{HH} 1$ for QW1 and $\mathrm{HH} 2$ for QW2). The position of the lowest subband energy levels are marked with bold solid lines in each QW, and the corresponding electron and heavy hole wavefunctions are plotted with solid curves for the states confined to QW1 at zero electric field, and dashed curves for the states confined to QW2 at zero electric field. The Coulomb interactions have been ignored in the calculation of these wavefunctions. For a barrier of $50 \AA$, this is a good approxmation, since the magnitudes of the electron wavefunctions in Fig. 2 are altered less than $4 \%$, even at resonance when the Coulomb interaction is included. For wider barriers, the Coulomb interaction increases and the analysis in the following sections will include this effect.

It is noted that the electron wavefunctions are modified substantially when the electric field is increased from 0 to $70 \mathrm{kV} / \mathrm{cm}$, and there is a strong coupling between the electron states in the two QW's. The heavy hole wavefunctions experience no noticeable change due to a much stronger confinement, and the coupling between the heavy holes states is negligible. At $F=0$, electron state $E 2$ is confined to QW2 and electron state $E 1$ is confined to QW1. At $F=35 \mathrm{kV} / \mathrm{cm}$, the electron subbands of the two QW's are in resonance and the wavefunctions are approximately equally distributed in both QW's. When $F$ is increased to $F=70 \mathrm{kV} / \mathrm{cm}$, the subband energies from the two QW's are now largely separated and $E 1$ is confined to QW2 and E2 is confined to QW1. The interchange of the electron wavefunctions between the two QW's involves an anticrossing [5], [6] of the energy levels, i.e., a minimum level separation of $E 1$ and $E 2$.

The coupling has an appreciable effect on the excitons. In Fig. 2(a) where $F=0$, the binding energies for the direct excitons corresponding to bound electron-hole pairs of $\mathrm{HH} 2-$ $E 2$ and $\mathrm{HH} 1-E 1$ are strong, due to a large overlap between the wavefunctions. The corresponding exciton oscillator strengths are, therefore, large. The binding energies for the indirect excitons corresponding to bound electron-hole pairs of $\mathrm{HH} 1$ $E 2$ and $\mathrm{HH} 2-E 1$ are small because the overlaps between the wavefunctions belonging to these excitons are small (see Fig. 2(a)). The corresponding exciton oscillator strengths are, therefore, also small. When the electric field is increased to resonance $F=35 \mathrm{kV} / \mathrm{cm}$ (see Fig. 2(b), the binding energies and oscillator strengths for the direct excitons are reduced, due to a delocalization of the electron wavefunctions. In Fig. 2(c) the electric field is further increased to $F=70$ $\mathrm{kV} / \mathrm{cm}$ and the electron wavefunctions are localized again, but in the opposite QW's compared to Fig. 2(a). Now the binding energies and oscillator strengths for the HH1-E2 exciton and the HH2-E1 exciton are strong (large overlap between the wavefunctions) and the binding energies and oscillator strengths for the $\mathrm{HH} 1-E 1$ exciton and $\mathrm{HH} 2-E 2$ exciton are weak (small overlap between the wavefunctions). The physical mechanism is thus a reduction in the exciton binding energies and oscillator strengths by resonant coupling between two quantum wells. The reduction in the exciton oscillator strengths causes a similar reduction in the absorption coefficient near the absorption edge. The detailed change in the absorption can be calculated using a computer model, which is described in the following section.

\section{MODELING OF EXCITONS IN COUPLED ASYMMETRIC QUANTUM WELLS}

\section{A. The Method}

A variational model of excitons in coupled symmetric QW's has been reported by Fox et al. [5]-[7]. A similar procedure will be used here for asymmetric QW's, and only a brief description of the model based on the effective mass approximation will, therefore, be presented. With the Coulomb interaction set to zero, the electron and hole envelope wavefunctions in the coupled QW's can be determined using a tunnel resonance method [11]. The following trial exciton envelope wavefunction can be used for an exciton formed between an $i$ th electron state and a $j$ th hole state

$$
\begin{aligned}
\Psi_{i j}^{(\mathrm{var})}\left(\rho, z_{e}, z_{h}\right) & =\phi_{e i}\left(z_{e}\right) f_{h j}\left(z_{h}\right) \psi_{1 s}^{i j}(\rho) \\
\phi_{e i}\left(z_{e}\right) & =\sum_{n=1}^{2} c_{\mathrm{in}} f_{e n}\left(z_{e}\right)
\end{aligned}
$$

where $z_{e}\left(z_{h}\right)$ is the position of the electron (hole) in the direction perpendicular to the QW's, $\rho$ is the inplane $(x y)$ 
electron-hole separation, $\rho=\sqrt{x^{2}+y^{2}} ; f_{e n}\left(z_{e}\right)$, and $f_{h j}\left(z_{h}\right)$ are the electron and hole envelope functions assuming no Coulomb interaction; $\phi_{e i}\left(z_{e}\right)$ is the modified electron wavefunction due to the Coulomb interaction and $c_{\text {in }}$ are the variable coupling coefficients. The holes are strongly localized due to a larger effective mass and a larger band offset; the coupling between them is small and will be neglected. The coupling between the hole subbands in each individual $\mathrm{QW}$ is also neglected here.

$\psi_{1 s}^{i j}(\rho)$ is a $1 s$ like two-dimensional trial wavefunction [11]

$$
\psi_{1 s}^{i j}(\rho)=\left(\frac{2}{\pi}\right)^{1 / 2} \frac{1}{\lambda_{i j}} \exp \left(\frac{-\rho}{\lambda_{i j}}\right)
$$

where $\lambda_{i j}$ is a parameter to be determined variationally.

The exciton binding energy for each exciton formed between an $i$ th electron subband and a $j$ th hole subband is determined by finding the lowest eigenvalue of the exciton Hamiltonian $H$

$$
E_{\min , i j}=\left\langle\psi_{i j}^{(\operatorname{var})}|H| \psi_{i j}^{(\mathrm{var})}\right\rangle .
$$

This is performed first for $i=1$ by varying the variational parameter $\lambda_{1 j}$ and the coupling coefficient $c_{11} c_{12}$ being determined by requiring that the wavefunction is normalized. The two other coupling coefficients related to $i=2, c_{21}$, and $c_{22}$ are determined by requiring normalization and that the two electron wavefunctions $\left(c_{11} f_{e 1}+c_{12} f_{e 2}\right)$ and $\left(c_{21} f_{e 1}+c_{22} f_{e 2}\right)$ are orthogonal. Now, with the coupling coefficients given, the exciton binding energy for $i=2$ is determined by varying the parameter $\lambda_{2 j}$. This procedure is carried out for each $j$ corresponding to the heavy hole and light hole subband states. The exciton binding energies $E_{x, i j}$ are then given by

$$
E_{x, i j}=E_{i}+E_{j}-E_{\min , i j},
$$

$E_{i}$, and $E_{j}$ being the electron and hole energies corresponding to the wavefunctions $\phi_{e i}\left(z_{e}\right)$ and $f_{h j}\left(z_{h}\right)$, respectively.

The absorption coefficient for the discrete exciton states is calculated using [12]

$$
\begin{aligned}
\alpha_{\mathrm{ex}}(\hbar \omega) & \\
= & \frac{4 \pi^{2} e^{2} \hbar}{n_{0} m_{0}^{2} c \hbar \omega\left(L_{1}+L_{2}\right)} \sum_{i, j} \frac{4}{\pi \lambda_{i j}^{2}}\left|\int d z \phi_{e i}\left(z_{e}\right)^{*} f_{h j}\left(z_{h}\right)\right|^{2} \\
& \times|\langle j|\bar{\varepsilon} \cdot \bar{p}| i\rangle|^{2} \delta\left(\hbar \omega-E_{\min , i, j}-E_{g}\right)
\end{aligned}
$$

where $n_{0}$ is the refractive index, $m_{0}$ is the free electron mass, $c$ is the velocity of light in vacuum, $\hbar \omega$ is the photon energy, and $L_{1}$ and $L_{2}$ are the quantum well widths of QW1 and QW2, respectively, $\langle j|\bar{\varepsilon} \cdot \bar{p}| i\rangle$ is the optical matrix element between the rapid varying Bloch functions, $E_{g}$ is the energy gap of the bulk semiconductor, and $\delta\left(\hbar \omega-E_{\min , i, j}-E_{g}\right)$ is the delta function. The nonvanishing optical matrix elements for the polarization $p_{x}$ along the plane of the QW's [transverse electric (TE) polarization] and the polarization $p_{z}$ perpendicular to the plane of the QW's [transverse magnetic (TM) polarization] can be determined from symmetry considerations (e.g., [13]).

For the exciton continuum states, the following expression for two-dimensional excitons is used [14]

$$
\begin{aligned}
\alpha_{c}(\hbar \omega)= & \sum_{i j} \frac{4 \pi^{2} e^{2} \hbar}{n_{0} m_{0}^{2} c\left(L_{1}+L_{2}\right) \hbar \omega} \frac{e^{\pi a}}{\cosh (\pi a)} \\
& \times\left|\bar{\varepsilon} \cdot \bar{p}_{i j}(\overline{0})\right|^{2} J_{i j}(\hbar \omega), \\
a= & \sqrt{\frac{R_{y}}{\hbar \omega-E_{i}-E_{j}}}
\end{aligned}
$$

where $R_{y}$ is the exciton Rydberg, $R_{y}=\frac{e^{4} \mu_{\|}}{2 \varepsilon_{0}^{2} \hbar^{2}}, \mu_{\|}$being the in-plane effective mass of the exciton, $\bar{p}_{i j}(\overline{0})$ is the optical matrix element between the $i$ th subband electron state, and the $j$ th subband hole state at the center of the first Brioullin zone

$$
\bar{p}_{i j}(\overline{0})=\langle j|\bar{p}| i\rangle \int d z \phi_{e i}(z) f_{h j}(z)
$$

and $J_{i j}(\hbar \omega)$ is the two dimensional density of states, which for parabolic bands is giving by

$$
J_{i j}(\hbar \omega)=\frac{\mu_{\|}}{\hbar^{2} \pi} \theta\left[\hbar \omega-E_{i}-E_{j}\right]
$$

where $\theta\left[\hbar \omega-E_{i}-E_{j}\right]$ is the unity step function. To take account of broadening due to phonons, impurity scattering, electric field inhomogeneities, etc., the delta function in (6) and the unity step function in (9) can readily be folded by Gaussian or Lorentzian functions. We use Gaussian functions which tend to model experimental results better [15] and use the experimental data of the line widths $\Gamma_{X}$ from [16] $(\sim 10 \mathrm{meV}$ at room temperature). This model has yielded good agreement to experimental results of absorption spectra of GaAs-GaAlAs single quantum well structures as a function of the electric field [17].

\section{B. Results}

Using the model described in the previous section, the electrooptical properties of a GaInAs-InP coupled asymmetric QW structure can be determined. The material parameters used in the model are depicted in Table I, and it is assumed that the light is TE-polarized. The following calculations are performed on a $30-\AA$ GaInAs quantum well (QW1) and a $40-$ $\AA$ GaInAs quantum well (QW2), separated by a $50-\AA$ or $70-\AA$ InP barrier. Fig. 3 shows the results of the position of (a) the four heavy hole exciton transitions with lowest energy, $\mathrm{HH} j$ $E i$, and (b) the four light hole exciton transitions with lowest energy, $\mathrm{LH} j-E i$, where the solid curves are for a $50-\AA$ InP barrier and the dashed curves are for a $70-\AA$ InP barrier. The notation for the electron and hole subbands is the same as in Fig. 2. The transition wavelength is defined as

$$
\lambda\left(\mathrm{HH}_{j}-E i\right)=h c /\left(E_{\min , i j}+E_{g}\right)
$$

where $E_{\min , i j}$ is the exciton eigenvalue determined variationally from (5) and $E_{g}$ is the energy gap of GaInAs. The HH1-E 1 exciton is direct at low electric fields and it is positioned at the longest transition wavelength. At a resonant electric field of $F_{0} \cong 35 \mathrm{kV} / \mathrm{cm}$ for the $50-\AA$ InP barrier and $F_{0} \cong 30 \mathrm{kV} / \mathrm{cm}$ for the $70-\AA \mathrm{InP}$ barrier, anticrossing (see Section III) occurs between the HH1-E1 excitonic transition, which is direct below resonance and indirect above resonance, 


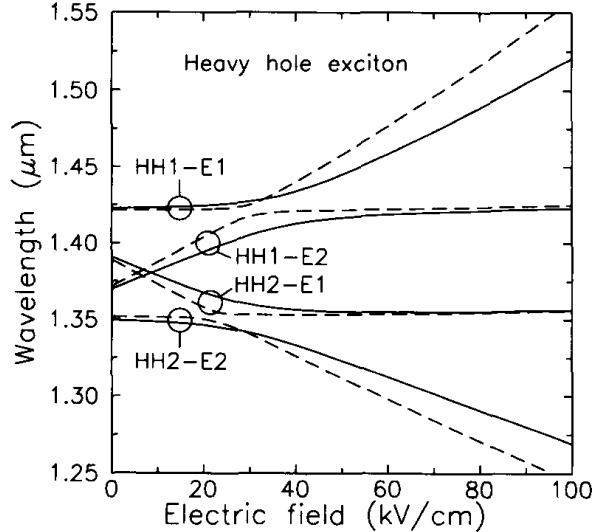

(a)

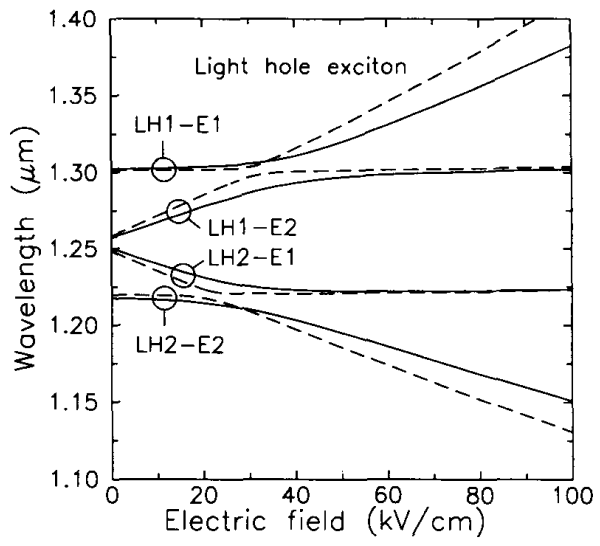

(b)

Fig. 3. Calculations of the transition wavelength for (a) the four heavy hole excitons with lowest energy, $\mathrm{HH} j-\mathrm{E} i$ and (b) the four light hole excitons with lowest energy, $\mathrm{LH} j-\mathrm{E} i$ in a $30-\AA / 40-\AA$ GaInAs coupled quantum well structure with a $50-\AA$ InP barrier (solid curves) and a $70-\AA \AA$ InP barrier (dashed curves) as function of electric field. The model used is described in the text and the notation for the electron subbands $E i$ and the hole subbands $\mathrm{HH} j$ $(\mathrm{LH} j)$ is the same as in Fig. 2.

and the HH1-E2 excitonic transition, which is indirect below resonance and direct above resonance. This means that below resonance, the $\mathrm{HH} 1-E 1$ excitonic transition is strong (large oscillator strength) and dominates the absorption near $\lambda \sim 1.42$ $\mu \mathrm{m}$ (see Fig. 3(a)), but above resonance the transition becomes weak. The opposite is the case for the HH1-E2 transition. The transition is weak below resonance, and above resonance it dominates the absorption near $\lambda \sim 1.42 \mu \mathrm{m}$.

Similarly, anticrossing also occurs between the HH2-E2 exciton and the HH2-E1 exciton. However, this takes place at a lower resonant electric field of $F_{0} \cong 32 \mathrm{kV} / \mathrm{cm}$ for the $50-\AA$ InP barrier and $F_{0} \cong 23 \mathrm{kV} / \mathrm{cm}$ for the $70-\AA$ InP barrier. The different value of $F_{0}$ compared to the case of anticrossing between the HH1-E1 exciton and the HH1-E2 exciton is due to the Coulomb interaction between the excitons and the difference is larger the larger the barrier between the coupled QW's. The difference for the $50-\AA$ barrier is $\sim 35 \mathrm{kV} / \mathrm{cm}-32$ $\mathrm{kV} / \mathrm{cm}=3 \mathrm{kV} / \mathrm{cm}$, and for the $70-\AA$ barrier it is $\sim 30 \mathrm{kV} / \mathrm{cm}$
TABLE I

Material Constants Used IN the Model Described in the Tex

\begin{tabular}{|c|c|c|}
\hline & $\operatorname{InP}$ & GaInÀs \\
\hline Band gap $E_{g}[\mathrm{eV}]$ & 1.351 (a) & 0.74 (b) \\
\hline Refractive index $\mathrm{n}(1.55 \mu \mathrm{m}) \quad[1\}$ & 3.17 (c) & 3.65 (d) \\
\hline Electron effective mass $m_{e}\left[m_{0}\right]$ & 0.077 (e) & $0.041 \quad(e)$ \\
\hline Heavy hole effective mass $m_{\mathrm{hb}}\left[m_{0}\right]$ & 0.56 (f) & 0.50 (f) \\
\hline Light hole effective mass $m_{2 h}\left[m_{0}\right]$ & $0.12\langle\mathrm{f}\rangle$ & 0.051 (f) \\
\hline Dielectric constant $\varepsilon_{\mathrm{s}}\left[\varepsilon_{0}\right] *$, & $12.4(\mathrm{E})$ & $13.8(f)$ \\
\hline Band of fset $Q_{c} / Q_{v}\{z\}$ & \multicolumn{2}{|c|}{$40 / 60 \quad(g)$} \\
\hline $\begin{array}{l}\text { optical matrix element } \\
2 / \mathrm{m}_{0}\left|\left\langle j\left|p_{x}\right| i\right\rangle\right|^{2}[\mathrm{ev}]\end{array}$ & \multicolumn{2}{|c|}{$23.8(\mathrm{~h})^{\dagger}$} \\
\hline
\end{tabular}

*) dielectric constant at zero frequency.

t) linear interpolation of GaAs and InAs

(a) Semiconductors, Ed. O. Madelung, M. Schultz, and $K$. Weiss Landolt-Bernstein, New Series, Group 3, vol. 179 (Springer-Verlag, Berlin, 1982).

(b) D.K. Gaski1l, N. Bottka, I. Aina, and M. Mattingly, Appl. Phys. Lett., 56, p.1269 (1990)

(c) B. Broberg, and S. Lindgren, J. Appl. Phys., 55, p.3376 (1984)

(d) H. Burkhard, H.W. Dinges, and E. Kuphal, J. Appl. Phys., 53, p. 655 (1982).

(e) M.B. Panish, H. Temkin, R.A. Hamm, and S.N.G. Chu, Appl. Phys Lett., 49, p-164 (1986).

(f) "GaInAsP Alloy Semiconductors," Ed. T.P. Pearsall (John Wiley \& Sons, 1982)

(g) B.I. Miller, E.F. Schubert, U. Koren, A. Ourmazd, A.H. Dayem

and J. Capik, Appl. Physt Lett. $49,0.1384\{1986\}$

(h) P. Lawaetz, phys Rev, B, 19, p 3460 (1971).

- $23 \mathrm{kV} / \mathrm{cm}=7 \mathrm{kV} / \mathrm{cm}$. When the Coulomb interaction is neglected, the anticrossing occurs at the same electric field $F_{0}$ for all excitons, and the value of $F_{0}$ lies in between the two different resonant electric fields in Fig. 3. The light hole excitonic transitions (see Fig. 3(b)) exhibit a similar behavior.

The exciton binding energies are shown for Fig. 4(a), the four heavy hole excitons with lowest energy, and Fig. 4(b), the four light hole excitons with lowest energy. The solid curves are for the $50-\AA$ barrier and the dashed curves are for the $70-\AA$ barrier. The different binding energies are indexed as in Fig. 3. The excitons $\mathrm{HH} 1-E 1$ and $\mathrm{HH} 2-E 2$, which are direct below resonance, have large binding energies at low electric fields and small binding energies at large electric fields where they become indirect. The opposite is the case for the excitons $H H 1-E 2$ and $H H 2-E 1$ which are indirect below resonance and direct above resonance. It is noted that the transition between high and low binding energy occurs at lower electric fields for the $70-\AA$ barrier (dashed curves) than for the $50-\AA$ barriers (solid curves), which is due to the fact that the coupling and therefore, the splitting between the subband energies decreases with increasing barriers. The transition for the $70-\AA$ barrier is also sharper because the tunneling time from one $\mathrm{QW}$ to the other increases with increasing barriers [18], implying that the broadening of the energy levels decreases with increasing barriers.

Using (6) and (7), the calculated absorption spectra for the $30-\AA / 40-\AA$ coupled $Q W ' s$ with a $50-\AA$ barrier are depicted in Fig. 5(a) as function of wavelength for an electric field 


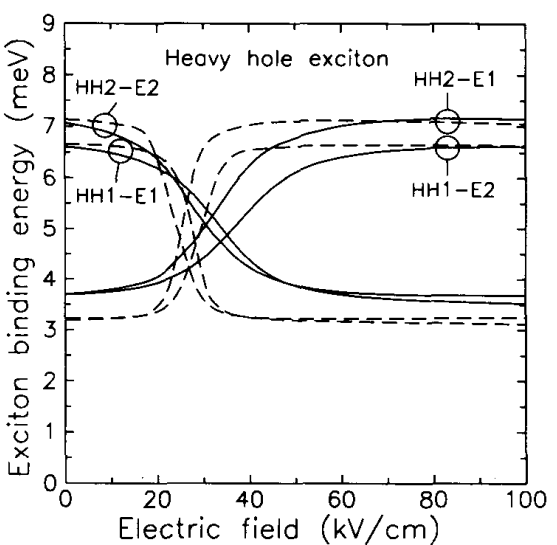

(a)

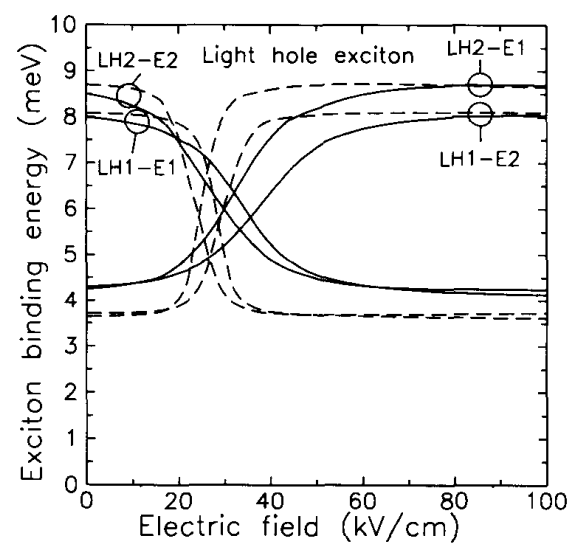

(b)

Fig. 4. Calculations of the binding energies for (a) the four heavy hole excitons with lowest energy, $\mathrm{HH} j-\mathrm{E} i$ and (b) the four light hole excitons with lowest energy, $\mathrm{LH} j-\mathrm{E} i$ in a $30-\AA / 40-\AA$ GaInAs coupled quantum well structure with a $50-\AA$ InP barrier (solid curves) and a $70-\AA$ InP barrier (dashed curves) as a function of the electric field.

of $F=0 \mathrm{kV} / \mathrm{cm}$ (solid curve), $F=35 \mathrm{kV} / \mathrm{cm}$ (dashed curve), and $F=70 \mathrm{kV} / \mathrm{cm}$ (dashdotted curve). The positions of the dominant excitonic transitions are indicated. At $F=$ 0 , the HH1-E1 exciton is direct and the HH1-E2 exciton is indirect and the exciton peak at $\lambda \sim 1.42 \mu \mathrm{m}$ is therefore dominated by the HH1-E1 exciton. At resonance $F=35$ $\mathrm{kV} / \mathrm{cm}$, both excitons contribute to the absorption process, but due to the smaller overlaps between the electron and the hole wavefunctions at resonance (see Fig. 2), the delocalization of the excitons results in a reduced absorption near $1.42 \mu \mathrm{m}$. A $F=70 \mathrm{kV} / \mathrm{cm}$, the $\mathrm{HH} 1-E 1$ exciton is indirect and the peak at $\lambda \sim 1.42 \mu \mathrm{m}$ is now dominated by the HH1-E2 exciton, which has become direct. A similar behavior is observed for the other dominant excitonic transitions $\mathrm{HH} 2-E 2 / \mathrm{HH} 2-E 1$, LH1-E1/LH1-E2 and LH2-E2/LH2-E1 (see Fig. 5(a)).

It is observed that the HH1-E1/HH1-E2 peak in the absorption spectrum (see Fig. 5(a)) is only shifted slightly in wavelength and the peak nearly recovers to the same strength as at zero electric field. This is consistent with the fact that according to Figs. 3(a) and 4(a), there is only a small

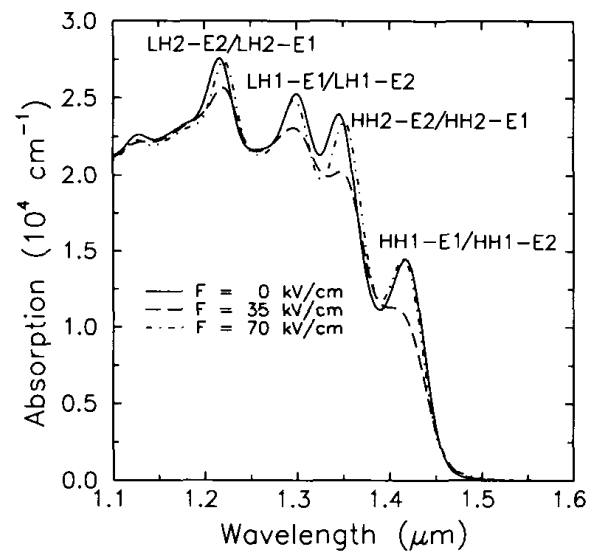

(a)

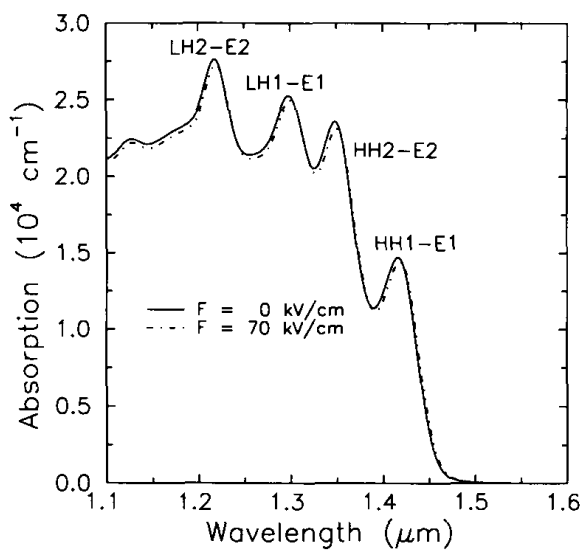

(b)

Fig. 5. Calculated absorption spectra for a $30-\AA / 40-\AA$ GaInAs coupled quantum well structure with (a) a $50-\AA$ InP barrier and (b) an infinite wide InP barrier as function of wavelength for an applied electric field of $F=0 \mathrm{kV} / \mathrm{cm}$ (solid curve), $F=35 \mathrm{kV} / \mathrm{cm}$ (dashed curve, not shown in (b)) and $F=70$ $\mathrm{kV} / \mathrm{cm}$ (dashdotted curve). The position of the dominant excitonic transitions (see Figs. 3 and 4) are indicated. In (b) there are only direct transitions.

difference in the positions and binding energies of the HH1$E 1$ exciton below resonance and the HH1- $E 2$ exciton above resonance. The exciton peaks belonging to $\mathrm{HH} 1-E 1 / \mathrm{HH} 1-$ $E 2$ and LH1-E1/LH1-E2, however, experience a slight blue shift, whilst the exciton peaks belonging to $\mathrm{HH} 2-E 2 / \mathrm{HH} 2-E 1$, and $\mathrm{LH} 2-E 2 / \mathrm{LH} 2-E 1$ experience a slight red shift when the electric field is increased from $F=0$ to $F=70 \mathrm{kV} / \mathrm{cm}$. This is a consequence of the Coulomb interaction.

For comparison, Fig. 5(b) shows the similar absorption spectra for $30-\AA / 40-\AA$ uncoupled QW's (infinite barriers) for $F=0 \mathrm{kV} / \mathrm{cm}$ (solid curve) and $F=70 \mathrm{kV} / \mathrm{cm}$ (dashdotted curve). In this case, the absorption spectra were obtained by performing calculations for 30 and $40-\AA$ single $Q W^{\prime}$ 's and adding the contributions from the two QW's. The result shows the influence of the QCSE and it is observed to be small compared to the effect of excitonic delocalization.

Performing the Kramers-Kronig transform (1) on the results from Fig. 5 , yields the change in the refractive index $\Delta n$ for $F=35$ and $70 \mathrm{kV} / \mathrm{cm}$ in Fig. 6, where the InP barrier is $50 \AA$ 


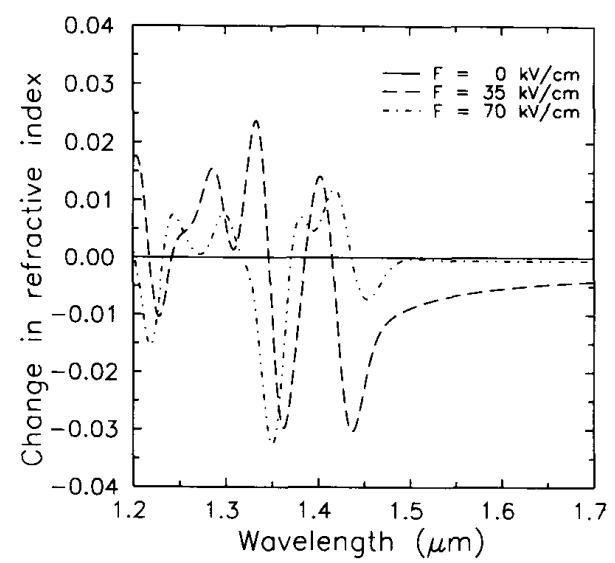

(a)

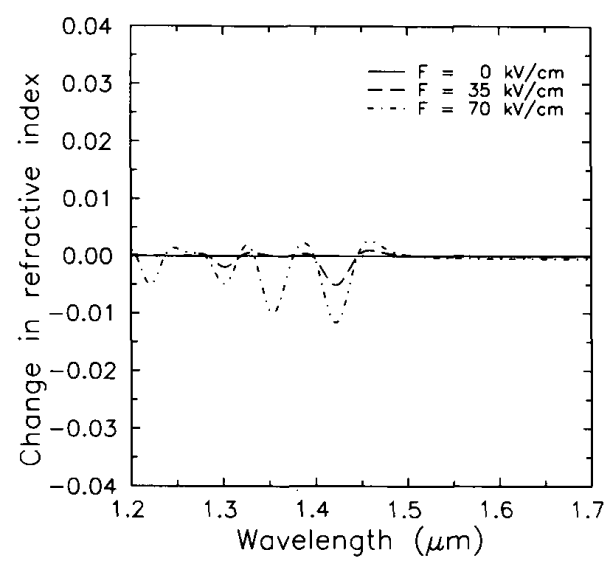

(b)

Fig. 6. Calculated change in refractive index for a $30-\AA / 40-\AA$ GaInAs coupled quantum well structure with (a) a $50-\AA$ InP barrier and (b) an infinite wide InP barrier as function of wavelength for an applied electric field of $F=$ $35 \mathrm{kV} / \mathrm{cm}$ (dashed curve) and $F=70 \mathrm{kV} / \mathrm{cm}$ (dashdotted curve). The results are obtained by performing the Kramers-Kronig transform on the calculated absorption spectra shown in Fig. 5.

in Fig. 6(a), and infinite in Fig. 6(b). In Fig. 6(a), for $F=35$ $\mathrm{kV} / \mathrm{cm}$ a $\Delta n$ of $7.5 \times 10^{-3}$ is obtained at $1.55 \mu \mathrm{m}$ where the absorption coefficient is small $\left(\sim 5 \mathrm{~cm}^{-1}\right)$. The corresponding change for the uncoupled configuration is more than one order of magnitude smaller: $\Delta n=3.8 \times 10^{-4}$ for $F=70 \mathrm{kV} / \mathrm{cm}$ and even less for $F=35 \mathrm{kV} / \mathrm{cm}$.

Fig. 7 shows the calculated $\Delta n$ at $\lambda=1.55 \mu \mathrm{m}$ as a function of the electric field for the $30-\AA / 40-\AA$ GaInAs coupled QW configuration with an InP barrier of $30 \AA$ (dashed curve), $50 \AA$ (solid curve), and $70 \AA$ (dotted curve) and for the case of no coupling between the QW's (dashdotted curve). For the coupled QW's, $\Delta n$ decreases with increasing electric field until resonance, whereupon $\Delta n$ increases toward zero. The resonance widths become sharper and the resonance peak moves toward lower electric field for increasing barrier width. This feature is also observed in Figs. 3 and 4 for the position of the excitonic transitions and for the exciton binding energies, respectively. It is interesting to note that for the $70-\AA$ barrier,

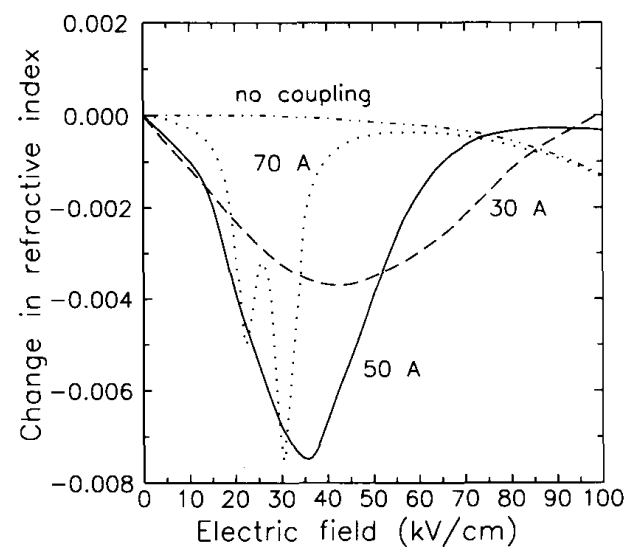

Fig. 7. Calculated change in the refractive index at a wavelength of $\lambda=$ $1.55 \mu \mathrm{m}$ for a $30-\AA / 40-\AA$ GaInAs coupled quantum well structure with a $30-\AA$ InP barrier (dashed curve), a $50-\AA$ InP barrier (solid curve), a $70-\AA$ InP barrier (dotted curve), and an infinite wide InP barrier (dashdotted curve) as a function of the electric field.

two resonances occur due to the excitonic splitting. For smaller barriers, the broadening is large and the splitting is too small to be resolved. At small and large electric fields, the result for the 70- $\AA$ barrier approaches the result for the QW's with no coupling. Fig. 7 clearly shows that there is a considerable enhancement to $\Delta n$ from the effect of delocalized excitons compared to the QCSE.

\section{UTILIZATION OF COUPLED EXCITONS in Optoelectronic Devices}

\section{A. Electrooptical Modulation of the Refractive Index}

The strong electrorefractive effect in GaInAs-InP coupled asymmetric quantum wells predicted by the model, could be utilized in optical waveguide devices based on optical phase modulation. The electrorefractive response can be defined as

$$
\begin{aligned}
R_{n} & =\Gamma_{C} \Delta \phi /(V L)=\Gamma_{C}\left(\frac{360^{\circ}}{\lambda} \Delta n L\right) /(F d L) \\
& =\Gamma_{C} \frac{360^{\circ} \Delta n}{\lambda F d}
\end{aligned}
$$

where $\Gamma_{C}$ is the confinement factor of the guided optical mode, $\Delta \phi=\frac{360^{\circ}}{\lambda} \Delta n$ is the phase change of the light, $L$ is the length of the waveguide, and $V=F d$ is the applied voltage necessary for a $\Delta \phi$ phase change, $d$ being the height of the $\mathrm{QW}$-region. For a configuration with a $50-\AA \mathrm{InP}$ barrier, a $\Delta n=7.5 \times 10^{-3}$ is obtained according to Fig. 7 , when the electric field $F=35 \mathrm{kV} / \mathrm{cm}$. Assuming an optical waveguide comprising a $d=0.6 \mu \mathrm{m}$ wide coupled QW-region with a $50-\AA$ InP barrier, as analyzed in the previous section yields a confinement factor for the QW's of $\Gamma_{C}=0.34$. Inserting the above mentioned parameters in the expression for $R_{n}$, yields $R_{n}=282^{\circ} /(\mathrm{Vmm})$. Assuming uncoupled QW's and a change in the electric field from 0 to $70 \mathrm{kV} / \mathrm{cm}$, the similar result is $R_{n}=7.3^{\circ} /(\mathrm{Vmm})$. Experimentally, $15^{\circ} /(\mathrm{Vmm})$ has been reported for optical waveguides based on GaInAs-InP 
MQW structures comprising 55- $\AA$ wide GaInAs uncoupled QW's [19]. The coupled QW's are therefore expected to yield a large improvement in the electrooptical response.

\section{B. Self-Photo-Induced Modulation of the Refractive Index}

Connecting a pin diode waveguide comprising the GaInAs-InP coupled asymmetric QW structure to a constant voltage source $V$ in series with a resistor $R$, self-photoinduced phase modulation [20] could be obtained. A fraction of the light propagating in the waveguide is absorbed and creates electron-hole pairs which are swept out of the $i$-region of the pin diode to the neutral regions by the electric field. This causes a photocurrent flowing in the external circuit and a change in the voltage across the resistor. The subsequent voltage drop across the diode causes a change in the electric field across the QW's and thereby a change in the refractive index.

A simple analytical expression for the optical nonlinearity can be obtained as follows. Due to self-photo-induced modulation, the change in the electric field across the QW's as function of light intensity is given by [20]

$$
\Delta F=-\eta R \frac{q\left(1-e^{-\alpha_{0} \Gamma_{C} L}\right)}{\hbar \omega} W I_{\text {in }}
$$

where $\eta$ is the internal quantum efficiency, $W$ is the width of the waveguide, $\alpha_{0}$ is the absorption coefficient for the QW's, and $I_{\text {in }}$ is the input optical intensity. If the applied voltage has a magnitude corresponding to an operating point for zero optical intensity of $F_{0}=30 \mathrm{kV} / \mathrm{cm}$ in Fig. 7, then the change in refractive index is approximately linear in the change in electric field for values ranging from $30 \mathrm{kV} / \mathrm{cm}$ to $15 \mathrm{kV} / \mathrm{cm}$ (see Fig. 7)

$$
\Delta n=-a_{1} \Delta F
$$

where, according to Fig. 7 , the coefficient $a_{1} \cong 3.0 \times 10^{-7}$ $\mathrm{cm} / \mathrm{V}$ for $F=30 \mathrm{kV} / \mathrm{cm}$ and a $50-\AA$ InP barrier. The nonlinear coefficient $n_{2}$ can then be obtained from (10) and (11)

$$
n_{2}=\frac{\Delta n}{I_{\text {in }}}=\eta R \frac{q\left(1-e^{-\alpha L}\right)}{\hbar \omega} W \alpha_{1} .
$$

It is observed that $n_{2}$ can be enhanced by increasing the resistance of the bias circuit, and this has also been verified experimentally [20]. However, the enhancement is at expense of speed, due to an increased $\mathrm{RC}$ time constant. Assuming a wavelength of $1.55 \mu \mathrm{m}$, where $\alpha=\alpha_{0} \Gamma_{C} \cong 5 \times 0.34 \mathrm{~cm}^{-1}=$ $1.7 \mathrm{~cm}^{-1}$, and setting $R=1 \mathrm{k} \Omega, \eta=1, W=2 \mu \mathrm{m}, L=0.36$ $\mathrm{cm}$ in (12), yields $n_{2}=3.4 \times 10^{-8} \mathrm{~cm}^{2} / \mathrm{W}$. Using a relative dielectric constant $\varepsilon_{S}=13$ and $d=0.6 \mu \mathrm{m}$, the capacitance of the waveguide is

$$
C=\varepsilon_{S} \varepsilon_{0} \frac{W L}{d}=1.4 \times 10^{-12} \mathrm{~F}
$$

and the RC time constant is then $\tau_{\mathrm{RC}}=1.4 \mathrm{~ns}$.

A figure of merit for the optical nonlinearity is often defined as the optical nonlinearity divided by the product of the absorption and the time response. Using (12) and (13), and assuming that $\alpha_{0} \Gamma_{C} L \ll 1$, a simple expression can be obtained

$$
\frac{n_{2}}{\tau_{\mathrm{RC}}}=\eta a_{1} \frac{q d}{\varepsilon_{S} \varepsilon_{0} \hbar \omega}=20 \mathrm{~cm}^{3} / \mathrm{J}
$$

where the above mentioned parameters have been inserted in the last equality. This figure of merit can be compared to a figure of $0.1 \mathrm{~cm}^{3} / \mathrm{J}$ reported for GaAs-GaAlAs multiple quantum well waveguides [21] at a wavelength of $\lambda=870$ $\mathrm{nm}$ and $30 \mathrm{~cm}^{3} / \mathrm{J}$ for bulk InSb at a wavelength of $\lambda=5.4$ $\mu \mathrm{m}$ and at $77 \mathrm{~K}$ [22]. In both cases, the physical mechanism due to the nonlinearity were believed to be bandfilling. To the author's knowledge similar data are not available for GaInAs-InP MQW material. However, the figure of merit generally scales with the wavelength to the power of four, $n_{2} /(\alpha \tau) \propto \lambda^{4}$ [23]. Scaling the figure of merits at $870 \mathrm{~nm}$ and $5.4 \mu \mathrm{m}$ by $\lambda^{4}$ yields $1 \mathrm{~cm}^{3} / \mathrm{J}$ and $0.2 \mathrm{~cm}^{3} / \mathrm{J}$, respectively. This implies that the predicted value of $20 \mathrm{~cm}^{3} / \mathrm{J}$ at $1.55 \mu \mathrm{m}$ is large.

\section{CONCLUSION}

The effect of excitons in GaInAs-InP coupled asymmetric quantum wells on the refractive index modulation has been analyzed theoretically in this paper. A large modulation is obtained from excitonic coupling between a wider QW (40 $\AA$ ) and a narrower QW ( $30 \AA)$, both QW's being sufficiently narrow to result in a negligible quantum confined Stark effect. At low electric fields, the excitonic optical transition in the wide QW dominates the absorption near the absorption edge. When the electric field is increased to a value where the two wells couple strongly to each other, the excitons are delocalized and the absorption near the absorption edge is reduced. When the electric field is further increased, localization occurs again and the excitonic optical transition in the narrow QW now dominates the absorption near the absorption edge. As a consequence, the absorption spectrum nearly recovers to the absorption at zero electric field. Due to this effect, a large change in the refractive index can be obtained below the absorption edge. At resonance, the changes are maximum and sufficiently below or above resonance they are close to zero. Numerical calculations show that the changes in refractive index are more than one order of magnitude larger than for the quantum confined Stark effect in a similar QW structure, but with infinite barriers and no coupling between the QW's. In addition, it was shown that combining the effect of excitons in coupled asymmetric QW's and self-photo-induced modulation results in an optical nonlinearity with a figure of merit of $\sim 20 \mathrm{kV} / \mathrm{cm}$, which is predicted to be more than one order of magnitude larger than band edge resonance effects in III-V semiconductors.

\section{REFERENCES}

[1] J. E. Zucker, K. L. Jones, T, Y, Chang, N. Sauer, B. Tell, K. BrownGoebeler, M. Wegener, and D. S. Chemla, "Compact low-voltage InGaAs-InAlAs multiple quantum well waveguide interferometers," Electron. Lett., vol. 24, pp. 2029-2031, 1990.

[2] J. E. Zucker, T. L. Hendrickson, and C. A. Burrus, "Electrooptic phase modulation in GaAs-AlGaAs quantum well waveguides," Appl. Phys. Lett., vol. 52, pp. 945-947, 1988. 
[3] J. E. Zucker, K. L. Jones, T. Y. Chang, N. J. Sauer, M. D. Divino, and D. S. Chemla, "Multigigahertz bandwidth intensity modulators using tunable electron-density multiple quantum well waveguides," Appl. Phys. Lett., vol. 59, pp. 201-203, 1991.

[4] N. Susa, "Improvement in electroabsorption and the effects of parameter variations in the three step asymmetric coupled quantum well," J. Appl. Phys., vol. 73, pp. 932-942, 1993.

[5] A. M. Fox, D. A. B. Miller, G. Livescu, J. E. Cunningham, J. E. Henry, and W. Y. Jan, "Excitons in resonant coupling of quantum wells," Phvs. Rev. B, vol. 42 , pp. $1841-1844,1990$

[6] A. M. Fox, D. A. B. Miller, G. Livescu, J. E. Cunningham, and W. Y. Jan, "Excitonic effects in coupled quantum wells," Phys. Rev. B, vol. 44, pp. 6231-6242, 1991 .

[7] A. M. Fox, D. A. B. Miller, J. E. Cunningham, W. Y. Jan, C. Y. P. Chao, and S. L. Chuang, "Suppression of the observation of Stark ladders in optical measurements on superlattices by excitonic effects," Phys. Rev. $B$, vol. 46, pp. 15365-15375, 1992.

[8] G. H. Döhler, "Doping superlattices (" $n-i-p-i$ crystals")," J. Quantum. Electron., vol. QE-22, pp. 1682-1695, 1986.

[9] D. A. B. Miller, D. S. Chemla, T. C. Damen, A. C. Gossard, W Wiegmann, T. H. Wood, and C. A. Burrus, "Novel hybrid optically bistable switch: The quantum well self electrooptic effect device," Appl. Phys. Lett., vol. 45, pp. 13-15, 1984.

[10] F. Stern, "Dispersion of the index of refraction near the absorption edge of semiconductors," Phys. Rev., vol. 133, pp. A1653-A1664, 1964

[11] D. A. B. Miller, D. S. Chemla, T. C. Damen, A. C. Gossard, W Wiegmann, T. H. Wood, and C. A. Burrus, "Electric field dependence of optical absorption near the band gap of quantum well structures," Phys. Rev. B, vol. 32, pp. 1043-1060, 1985.

[12] F. Bassani and G. Pastori Parravicini, Electronic States and Optical Transitions in Solids. Oxford: Pergamon Press, 1975.

[13] G. D. Sanders and K. K. Bajaj, "Electronic properties and optical absorption spectra of $\mathrm{GaAs}-\mathrm{Al}_{x} \mathrm{Ga}_{1-x}$ As quantum wells in externally applied electric field," Phys. Rev. B, vol. 35, pp. 2308-2320, 1987.

[14] M. Shinada and S. Sugano, "Interband optical transitions in extremely anisotropic semiconductors. Part I: Bound and unbound exciton absorption," J. Phys. Soc. Jap., vol. 21, pp. 1936-1946, 1966.

[15] D. S. Chemla, D. A. B. Miller, P. W. Smith, A. C. Gossard, and
W. Wiegmann, "Room temperature excitonic nonlinear absorption and refraction in GaAs-AlGaAs multiple quantum well structures," J. Quantum Electron., vol. QE-22, pp. 265-275, 1984.

[16] Y. Kawaguchi and H. Asahi, "High temperature observation of heavy hole and light hole excitons in InGaAs-InP multiple quantum well structures grown by metalorganic molecular beam epitaxy," Appl. Phys. Lett., vol. 50, pp. 1243-1245, 1987.

[17] C. Thirstrup, "Optical nonlinearities in GaAs-GaAlAs multiple quantum well hetero nipi waveguides," Ph.D. thesis, Technical Univ. of Denmark, 1990.

[18] A. Hernández-Cabrera, P. Aceituno, and H. Cruz, "Self consistent calculations of coherent tunneling oscillations in asymmetric double quantum wells," Superlattices and Microstructures, vol. 111, pp. 375-382, 1992.

[19] C. Thirstrup, S. W. Pang, O. Albrektsen, and J. Hanberg, "Effects of reactive ion etching on optical and electrooptical properties of GaInAs-InP based strip loaded waveguides," J. Vac. Sci. Technol. B, vol. 11, pp. 1214-1221, 1993

[20] C. Thirstrup, P. Li Kam Wa, M. A. Pate, J. S. Roberts, and P. N. Robson, "Self-photo-induced phase modulation in GaAs-AlGaAs multiple quantum well waveguides," Electron. Lett., vol. 25, pp. 1668-1669. 1989 .

[21] C. Thirstrup, P. N. Robson, P. Li Kam Wa, M. A. Pate, C. C. Button, and J. S. Roberts, "Optical nonlinearities in GaAs-GaAlAs multiple quantum well hetero-nipi waveguides," J. Quantum Electron., vol. 28, pp. 864-874, 1992

[22] D. A. B. Miller, C. T. Seaton, M. E. Prise, and S. D. Smith, "Bandgap-resonant nonlinear refraction in III-V semiconductors," Phys. Rev. Lett., vol. 47, pp. 197-200, 1981.

[23] K. Watanabe and Y. Yamamoto, "Limits on tradeoffs between third order optical nonlinearity, absorption loss, and pulse duration in self induced transparency and real excitation," Phys. Rev. A, vol. 42, pp. 1699-1702, 1990.

Carsten Thirstrup, photograph and biography not available at the time of publication. 